\title{
A high accuracy numerical method based on spectral theory of compact operator for biharmonic eigenvalue equations
}

\section{Zhendong Luo*}

\section{"Correspondence:}

zhdluo@ncepu.edu.cn

School of Mathematics and Physics,

North China Electric Power

University, No. 2, Bei Nong Road,

Changping District, Beijing, 102206,

China

\section{Springer}

\begin{abstract}
In this study, a high accuracy numerical method based on the spectral theory of compact operator for biharmonic eigenvalue equations on a spherical domain is developed. By employing the orthogonal spherical polynomials approximation and the spectral theory of compact operator, the error estimates of approximate eigenvalues and eigenfunctions are provided. By adopting orthogonal spherical base functions, the discrete model with sparse mass and stiff matrices is established so that it is very efficient for finding the numerical solutions of biharmonic eigenvalue equations on the spherical domain. Some numerical examples are provided to validate the theoretical results.
\end{abstract}

MSC: $65 \mathrm{~N} 35 ; 65 \mathrm{~N} 30$

Keywords: biharmonic eigenvalue equations; spectral-Galerkin discretization; error estimates; spherical domain

\section{Introduction}

In this article, we consider the following biharmonic eigenvalue equations:

$$
\begin{aligned}
& \Delta^{2} u=\lambda u, \quad \text { in } \Omega, \\
& u=\frac{\partial u}{\partial \mathbf{n}}=0, \quad \text { on } \partial \Omega,
\end{aligned}
$$

where $\Omega \subset \mathbf{R}^{d}(d=2,3)$ is an open disk or a ball, $\frac{\partial u}{\partial \mathbf{n}}$ denotes the outer normal derivative of $u$ on $\partial \Omega$.

The biharmonic eigenvalue equations are used to describe the vibration and buckling of plates in mechanics (see, e.g., [1-3]) and transmission eigenvalue problem in inverse scattering theory (see, e.g., $[4,5])$. Therefore, the biharmonic eigenvalue equations have very wide extensive applications.

However, most of the existing work were concerned with the second-order elliptic eigenvalue problems and there are relatively few articles treating the biharmonic eigenvalue problems. In recent years, the numerical methods for the biharmonic eigenvalue problems adopted mainly the conforming finite element method (see [6-9]), the nonconforming finite element method (see [3, 10-12]), and the mixed finite element method (see [13-16]).

(c) 2016 Luo. This article is distributed under the terms of the Creative Commons Attribution 4.0 International License (http://creativecommons.org/licenses/by/4.0/), which permits unrestricted use, distribution, and reproduction in any medium, provided you give appropriate credit to the original author(s) and the source, provide a link to the Creative Commons license, and indicate if changes were made. 
For the conforming finite elements method, it requires globally continuously differentiable finite element spaces, therefore, they are difficult to construct and implement (in particular for three-dimensional problems). For the nonconforming finite element method, a disadvantage is that such elements do not come in a natural hierarchy and existing nonconforming elements only involve low-order polynomials so that they are not efficient for capturing smooth solutions. For the mixed finite element method, it can result in spurious solutions on non-convex domains for the boundary conditions of simply supported plates. In the last decade, the $C^{0}$ interior penalty Galerkin ( $C^{0}$ IPG) method had been developed for second-order elliptic eigenvalue problems (see [17-19]). Recently, Brenner et al. in [20] extended the $C^{0}$ IPG method to biharmonic eigenvalue problems and provided the method converges for all three types of boundary conditions (see [20]).

However, all methods mentioned above are low-order finite element methods so that it is very difficultly and expensively to obtain high accuracy numerical solutions, especially for the three-dimensional spherical domain. To the best of our knowledge, there is not any article on a high accuracy numerical method based on the spectral theory of compact operator for biharmonic eigenvalue equations on the spherical domain. Therefore, the task of this paper is to develop a high precision numerical method based on the spectral theory of compact operator for biharmonic eigenvalue equations in the spherical domain.

The rest of this paper is organized as follows. Section 2 provides some preliminaries. In Section 3, by employing the orthogonal spherical polynomials approximation and the spectral theory of compact operator, we derive the error estimates of approximate eigenvalues and eigenfunctions. In Section 4, by adopting orthogonal spherical base functions, we establish the discrete model with sparse mass and stiff matrices which is very efficient for finding the numerical solutions of biharmonic eigenvalue equations on the spherical domain. In Section 5, we provide some numerical examples to validate that the theoretical results are correct. Finally, we provide some conclusions in Section 6.

\section{Some preliminaries}

The Sobolev spaces and norms used in this paper are standard ([21]). For example, $H^{s}(\Omega)$ denotes the usual Sobolev space on $\Omega$ with real order $s$, equipped with the norm $\|\cdot\|_{s}$, and $H^{0}(\Omega)=L^{2}(\Omega), H_{0}^{2}(\Omega)=\left\{u \in H^{2}(\Omega): u=\frac{\partial u}{\partial \mathbf{n}}=0\right.$ on $\left.\partial \Omega\right\}$.

The unit circle and the unit sphere in $R^{d}$ are denoted, respectively, by

$$
\mathbf{B}^{d}:=\left\{\mathbf{x} \in R^{d}:\|\mathbf{x}\| \leq 1\right\} \quad \text { and } \quad \mathbb{S}^{d-1}:=\left\{\xi \in R^{d}:\|\boldsymbol{\xi}\|=1\right\}
$$

Moreover, we denote the sets of all nonnegative integers and all real numbers by $\mathbf{N}$ and $\mathbf{R}$, respectively. Further, for $n \in \mathbf{N}$, we denote the collection of all polynomials in $d$ variables with a total degree $\leq n$ by $\Pi_{n}^{d}$.

Let $\mathcal{H}_{n}^{d}$ denote the space of (solid) spherical harmonics of degree $n$ with $d$ variables, i.e.,

$$
\mathcal{H}_{n}^{d}=\left\{q \in \Pi_{n}^{d}: \Delta q=0 \text { and } q(\rho \mathbf{x})=\rho^{n} q(\mathbf{x}) \text { for any } \rho \in R \text { and } \mathbf{x} \in R^{d}\right\}
$$


It is easily known that

$$
a_{n}^{d}:=\operatorname{dim} \mathcal{H}_{n}^{d}=\left(\begin{array}{c}
n+d-1 \\
n
\end{array}\right)-\left(\begin{array}{c}
n+d-3 \\
n-2
\end{array}\right)= \begin{cases}2-\delta_{n, 0}, & d=2, \\
2 n+1, & d=3,\end{cases}
$$

where $\delta_{n, 0}$ is the Kronecker delta.

Let $\left\{Y_{\ell}^{n}: 1 \leq \ell \leq a_{n}^{d}\right\}$ be an orthonormal basis of $\mathcal{H}_{n}^{d}$ such that

$$
\int_{\mathbb{S}^{d-1}} Y_{\ell}^{n}(\xi) \overline{Y_{\ell^{\prime}}^{n^{\prime}}(\xi)} \mathrm{d} \omega=\omega_{d} \delta_{n, n^{\prime}} \delta_{\ell, \ell^{\prime}}, \quad 1 \leq \ell \leq a_{n}^{d}, 1 \leq \ell^{\prime} \leq a_{n^{\prime}}^{d}, n, n^{\prime} \geq 0
$$

where $\mathrm{d} \omega$ is the surface measure and $\omega_{d}=2 \pi^{\frac{d}{2}} / \Gamma\left(\frac{d}{2}\right)$ is the surface area.

Let $P_{j}^{(\mu, v)}(\zeta)$ denote the usual Jacobi polynomial of degree $j$ with respect to the weight function $(1-\zeta)^{\mu}(1+\zeta)^{v}$ on $[-1,1]$. Let $(a)_{n}:=a(a+1) \cdots(a+n-1)$ be the Pochhammer symbol. Define

$$
P_{j, \ell}^{\mu, n}(\mathbf{x}):=\frac{\left(n-j+\frac{d}{2}\right)_{j}}{\left(n-j+\frac{d}{2}+\mu\right)_{j}} P_{j}^{\left(\mu, n-2 j+\frac{d-2}{2}\right)}\left(2\|\mathbf{x}\|^{2}-1\right) Y_{\ell}^{n-2 j}(\mathbf{x})
$$

Then the set $\left\{P_{j, \ell}^{\mu, n}(\mathbf{x}): 0 \leq j \leq \frac{n}{2}, 1 \leq \ell \leq a_{n-2 j}^{d}\right\}$ is mutually orthogonal with respect to the weight function $\varpi_{\mu}(\mathbf{x})=(1-\|\mathbf{x}\|)^{\mu}$ whenever $\mu>-1$ (see [22]). More precisely,

$$
\int_{\mathbf{B}^{d}} P_{j, \ell}^{\mu, n}(\mathbf{x}) \overline{P_{j^{\prime}, \ell^{\prime}}^{\mu, n^{\prime}}(\mathbf{x})} \varpi_{\mu}(\mathbf{x}) \mathrm{d} \mathbf{x}=b_{d}^{\mu} h_{j, n}^{\mu} \delta_{n, n^{\prime}} \delta_{j, j^{\prime}} \delta_{\ell, \ell^{\prime}},
$$

where $b_{\mu}^{d}$ and $h_{j, n}^{\mu}$ are given by

$$
b_{d}^{\mu}:=\frac{\pi^{\frac{d}{2}} \Gamma(\mu+1)}{\Gamma\left(\mu+\frac{d}{2}+1\right)}, \quad h_{j, n}^{\mu}:=\frac{(\mu+1)_{j}\left(1-n-\frac{d}{2}\right)_{j}\left(\frac{d}{2}\right)_{n}}{j !\left(1-n-\frac{d}{2}-\mu\right)_{j}\left(\frac{d}{2}+\mu+1\right)_{n}} .
$$

For $f \in H_{0}^{s}\left(\mathbf{B}^{d}\right)$ with $s \in \mathbf{N}$, let $S_{N}^{-s} f$ denote its best polynomial approximation of degree at most $N$ by

$$
\left\langle\nabla^{s}\left(S_{N}^{-s} f-f\right), \nabla^{s} v\right\rangle=0, \quad v \in \Pi_{N}^{d} \cap H_{0}^{s}\left(\mathbf{B}^{d}\right),
$$

where $\nabla^{2 m}=\Delta^{m}$ and $\nabla^{2 m-1}=\nabla \Delta^{m-1}$ for any $m \in \mathbf{N}$. We have the following error estimate for $S_{N}^{-s}$ (see Theorem 4.3 in [22]).

Theorem 2.1 Let $r, s \in \mathbf{N}$ and $k \in \mathbf{N}_{0}$. If $\in H_{0}^{s}\left(\mathbf{B}^{d}\right) \cap H^{r}\left(\mathbf{B}^{d}\right)$ with $r \geq s$, then, for $N \geq s$,

$$
\left|f-S_{N}^{-s} f\right|_{H^{k}\left(\mathbf{B}^{d}\right)} \leq C N^{-r+k}\|f\|_{H^{r}\left(\mathbf{B}^{d}\right)}
$$

where $C$ used next is a positive constant which is possibly different at different occurrences, being independent of the degree $N$ of polynomials. 


\section{The operator formulations and error estimates}

\subsection{Operator formulations}

Put $V=H_{0}^{2}(\Omega)$. Then the weak form of (1)-(2) is given as follows.

Find $\lambda \in \mathbf{R}, 0 \neq u \in V$, such that

$$
a(u, v)=\lambda b(u, v), \quad \forall v \in V,
$$

where $a(u, v)=\int_{\Omega} \Delta u \Delta v \mathrm{~d} \mathbf{x}, b(u, v)=\int_{\Omega} u v \mathrm{~d} \mathbf{x}$.

The source problem associated with (5) is written as follows.

Find $u \in V$ such that

$$
a(u, v)=b(f, v), \quad \forall v \in V .
$$

It is obvious that $a(\cdot, \cdot)$ is a continuous, symmetric, and $V$-elliptic bilinear form on $V \times V$ and $b(f, \cdot)$ is a continuous and linear functional on $V$. Thus, we can use $\|\cdot\|_{a}=\sqrt{a(\cdot, \cdot)}$ as a norm in $V$ which is equivalent to the norm $\|\cdot\|_{2}$ induced by $H^{2}(\Omega)$ and we know from the Lax-Milgram theorem that (6) has a unique solution. Thus, according to the source problem (6), we define the operator $T: V \rightarrow V$ by

$$
a(T f, v)=b(f, v), \quad \forall v \in V .
$$

Thanks to [24], we know that (5) has the equivalent operator form

$$
T u=\frac{1}{\lambda} u
$$

For the above operator $T$, we have the following result.

Lemma 3.1 The operator $T: V \mapsto V$ is a self-adjoint compact one.

Proof For $\forall u, v \in V$, we have

$$
a(T u, v)=b(u, v)=b(v, u)=a(T v, u)=a(u, T v),
$$

Thus, $T: V \mapsto V$ is self-adjoint. By taking $f=u, v=T u$ in (7), we can obtain

$$
a(T u, T u)=b(u, T u) .
$$

From the Poincaré inequality, we can derive

$$
\gamma\|T u\|_{2}^{2} \leq a(T u, T u)=b(u, T u) \leq\|u\|_{0}\|T u\|_{0} \leq\|u\|_{0}\|T u\|_{2} .
$$

Thus, we have

$$
\|T u\|_{2} \leq \frac{1}{\gamma}\|u\|_{0}
$$

where $\gamma$ is a positive constant. 
Let $E$ be the bounded set in $V$. Since $V$ is compactly embedded in $L_{2}(\Omega)$, so $E$ is the sequentially compact set in $L^{2}(\Omega)$. From (9), we know that $T E$ is the sequentially compact set in $V$. Thus, $T: V \rightarrow V$ is a compact operator.

From the classical theory of abstract elliptic eigenvalue problem (see, e.g., [23, 24]), we know that all eigenvalues of $T$ are real and have finite algebraic multiplicity. We arrange the eigenvalues of $T$ by increasing order:

$$
0<\lambda_{1} \leq \lambda_{2} \leq \lambda_{3} \leq \cdots \nearrow+\infty
$$

Since the eigenfunctions corresponding to two arbitrary different eigenvalues of $T$ must be orthogonal, there must exist a standard orthogonal basis in eigenspace corresponding to the same eigenvalue. Thus, by using the eigenfunctions of $T$ corresponding to $\left\{\lambda_{j}\right\}$, we can construct a complete orthonormal system of $V$ as follows:

$$
u_{1}, u_{2}, \ldots, u_{j}, \ldots
$$

Let $X_{N}=\Pi_{N}^{d} \cap H_{0}^{2}(\Omega)$, then the spectral-Galerkin approximation of (5) is given as follows.

Find $\lambda_{N} \in \mathbf{R}, 0 \neq u_{N} \in X_{N}$ such that

$$
a\left(u_{N}, v_{N}\right)=\lambda_{N} b\left(u_{N}, v_{N}\right), \quad \forall v_{N} \in X_{N}
$$

The source problem associated with (10) is written as follows.

Find $u_{N} \in X_{N}$, such that

$$
a\left(u_{N}, v\right)=b(f, v), \quad \forall v \in X_{N}
$$

Likewise, we know from the Lax-Milgram theorem that (11) has a unique solution. Thus, we can define the operator $T_{N}: V \rightarrow X_{N}$ by

$$
a\left(T_{N} f, v\right)=b(f, v), \quad \forall v \in X_{N}
$$

From [24], we know that (10) has the equivalent operator form

$$
T_{N} u_{N}=\frac{1}{\lambda_{N}} u_{N}
$$

It is obvious that $T_{N}: V \rightarrow X_{N}$ is a finite rank operator.

Define the projection operator $\Pi_{N}^{2,0}: V \rightarrow X_{N}$ by

$$
a\left(u-\Pi_{N}^{2,0} u, v\right)=0, \quad \text { for all } u \in V, v \in X_{N} .
$$

We have the following result.

Lemma 3.2 Let $T$ and $T_{N}$ be linear bounded operator defined by (7) and (12), respectively. Then we have the following equality:

$$
T_{N}=\Pi_{N}^{2,0} T
$$


Proof For $\forall u \in V, v \in X_{N}$, we have

$$
a\left(\Pi_{N}^{2,0} T u-T_{N} u, v\right)=a\left(\Pi_{N}^{2,0} T u-T u, v\right)+a\left(T u-T_{N} u, v\right)=0 .
$$

By taking $v=\Pi_{N}^{2,0} T u-T_{N} u$ in (15), we can obtain

$$
a\left(\Pi_{N}^{2,0} T u-T_{N} u, \Pi_{N}^{2,0} T u-T_{N} u\right)=0 .
$$

Since $a(\cdot, \cdot)$ is V-elliptic, we can obtain

$$
T_{N}=\Pi_{N}^{2,0} T
$$

which completes the proof of Lemma 3.2.

It is obvious that

$$
\left.T_{N}\right|_{N}: X_{N} \rightarrow X_{N}
$$

is a self-adjoint finite rank operator and the eigenvalues of (10) can be arranged as

$$
0<\lambda_{1 N} \leq \lambda_{2 N} \leq \lambda_{3 N} \leq \cdots \lambda_{K N}, \quad K=\operatorname{dim}\left(X_{N}\right)
$$

\subsection{Error estimates}

In the following, we provide the error estimates. We first provide the following lemma.

Lemma 3.3 Let $(\lambda, u)$ and $\left(\lambda_{N}, u_{N}\right)$ be the eigenpair of (5) and (10), respectively. Then we have

$$
\lambda_{N}-\lambda=\frac{\left\|u_{N}-u\right\|_{a}^{2}}{\left\|u_{N}\right\|_{L^{2}(\Omega)}^{2}}-\lambda \frac{\left\|u_{N}-u\right\|_{L^{2}(\Omega)}^{2}}{\left\|u_{N}\right\|_{L^{2}(\Omega)}^{2}} .
$$

Proof From (5), we can derive

$$
\begin{aligned}
& a\left(u_{N}-u, u_{N}-u\right)-\lambda b\left(u_{N}-u, u_{N}-u\right) \\
& \quad=a\left(u_{N}, u_{N}\right)-2 a\left(u_{N}, u\right)+a(u, u)-\lambda b\left(u_{N}, u_{N}\right)+2 \lambda b\left(u_{N}, u\right)-\lambda b(u, u) \\
& \quad=a\left(u_{N}, u_{N}\right)-2 \lambda b\left(u_{N}, u\right)+\lambda b(u, u)-\lambda b\left(u_{N}, u_{N}\right)+2 \lambda b\left(u_{N}, u\right)-\lambda b(u, u) \\
& \quad=a\left(u_{N}, u_{N}\right)-\lambda b\left(u_{N}, u_{N}\right) .
\end{aligned}
$$

By dividing $b\left(u_{N}, u_{N}\right)$ and applying (10) to both sides of the above equation, we obtain

$$
\lambda_{N}-\lambda=\frac{\left\|u_{N}-u\right\|_{a}^{2}}{\left\|u_{N}\right\|_{L^{2}(\Omega)}^{2}}-\lambda \frac{\left\|u_{N}-u\right\|_{L^{2}(\Omega)}^{2}}{\left\|u_{N}\right\|_{L^{2}(\Omega)}^{2}},
$$

which completes the proof of Lemma 3.3. 
Put $\eta_{N}=\sup _{u \in V,\|u\|_{a}=1} \inf _{v \in X_{N}}\|T u-v\|_{a}$. It is clear that we have

$$
\eta_{N}=\sup _{u \in V,\|u\|_{a}=1} \inf _{v \in X_{N}}\|T u-v\|_{a} \rightarrow 0 \quad(N \rightarrow \infty)
$$

Thus, we have the following convergence on operators (also see the proof in [25]).

Theorem 3.1 We have

$$
\lim _{N \rightarrow \infty}\left\|T-T_{N}\right\|_{a}=0
$$

Proof By the definition of the operator norm, we have

$$
\begin{aligned}
\left\|T-T_{N}\right\|_{a} & =\sup _{u \in V,\|u\|_{a}=1}\left\|\left(T-T_{N}\right) u\right\|_{a}=\sup _{u \in V,\|u\|_{a}=1}\left\|T u-\Pi_{N}^{2,0} T u\right\|_{a} \\
& =\sup _{u \in V,\|u\|_{a}=1} \inf _{v \in X_{N}}\|T u-v\|_{a}=\eta_{N} .
\end{aligned}
$$

Then, from (18), we obtain the desired result.

Let $M(\lambda)$ denote the eigenfunctions space of (5) corresponding to the eigenvalue $\lambda$. We have the following results (also see the proof in [25]).

Theorem 3.2 Let $(\lambda, u)$ and $\left(\lambda_{N}, u_{N}\right)$ be the eigenpair of $(5)$ and (10), respectively. Then we have

$$
\begin{aligned}
& \left\|u-u_{N}\right\|_{a} \leq \sup _{u \in M(\lambda),\|u\|_{a}=1} \frac{C}{\lambda}\left\|u-\Pi_{N}^{2,0} u\right\|_{a}, \\
& \lambda_{N}-\lambda \leq \sup _{u \in M(\lambda),\|u\|_{a}=1} \frac{C}{\lambda^{2}} \frac{\left\|u-\Pi_{N}^{2,0} u\right\|_{a}^{2}}{\left\|u_{N}\right\|_{L^{2}(\Omega)}^{2}}
\end{aligned}
$$

Proof From Theorem 3.1, we know that $\left\|T-T_{N}\right\|_{a} \rightarrow 0(N \rightarrow \infty)$. Thus, according to Theorem 7.4 in [24], we have

$$
\left\|u-u_{N}\right\|_{a} \leq C\left\|\left.\left(T-T_{N}\right)\right|_{M(\lambda)}\right\|_{a} .
$$

Therefore, for any $u \in M(\lambda)$ satisfying $\|u\|_{a}=1$, we have

$$
\begin{aligned}
& \left\|\left(T-T_{N}\right) u\right\|_{a}=\left\|T u-\Pi_{N}^{2,0} T u\right\|_{a}=\frac{1}{\lambda}\left\|u-\Pi_{N}^{2,0} u\right\|_{a}, \\
& \left\|\left.\left(T-T_{N}\right)\right|_{M(\lambda)}\right\|_{a}=\sup _{u \in M(\lambda),\|u\|_{a}=1}\left\|\left(T-T_{N}\right) u\right\|_{a} .
\end{aligned}
$$

By combining (23) and (24) with (22), we get the desired result (20). By Lemma 3.3, we obtain

$$
\lambda_{N}-\lambda \leq \frac{\left\|u_{N}-u\right\|_{a}^{2}}{\left\|u_{N}\right\|_{L^{2}(\Omega)}^{2}},
$$

which together with (20) yields (21). 
Theorem 3.3 Let $(\lambda, u)$ and $\left(\lambda_{N}, u_{N}\right)$ be the eigenpair of (5) and (10), respectively. If $u \in$ $H_{0}^{2}(\Omega) \cap H^{r}(\Omega)$ with $r \geq 2$, then, for $N \geq 2$,

$$
\begin{aligned}
& \left\|u-u_{N}\right\|_{a} \leq \frac{C}{\lambda} N^{(2-r)} \sup _{u \in M(\lambda),\|u\|_{a}=1}\|u\|_{r}, \\
& \lambda_{N}-\lambda \leq \frac{C}{\lambda^{2}} N^{2(2-r)} \sup _{u \in M(\lambda),\|u\|_{a}=1} \frac{\|u\|_{r}^{2}}{\left\|u_{N}\right\|_{L^{2}(\Omega)}^{2}} .
\end{aligned}
$$

Proof From (14) and the continuity of $a(u, v)$, we have

$$
\begin{aligned}
\left\|u-\Pi_{N}^{2,0} u\right\|_{a}^{2} & =a\left(u-\Pi_{N}^{2,0} u, u-\Pi_{N}^{2,0} u\right) \\
& =\inf _{\phi_{N} \in X_{N}} a\left(u-\phi_{N}, u-\phi_{N}\right) \\
& \leq C \inf _{\phi_{N} \in X_{N}}\left|u-\phi_{N}\right|_{2}^{2} \\
& \leq C\left|u-S_{N}^{-2} u\right|_{2}^{2} .
\end{aligned}
$$

Thus, from Theorem 2.1, we can obtain

$$
\left\|u-\Pi_{N}^{2,0} u\right\|_{a}^{2} \leq C\left|u-S_{N}^{-2} u\right|_{2}^{2} \leq C N^{2(2-r)}\|u\|_{r}^{2} .
$$

By combing (26) with Theorem 3.2, we can get the desired result.

\section{Matrix formulation of the spectral-Galerkin approximation}

For $n \in \mathbf{N}, 2 \leq j \leq \frac{n}{2}$, and $1 \leq \ell \leq a_{n-2 j}^{d}$, put

$$
P_{j, \ell}^{-2, m}(\mathbf{x}):=\frac{\left(1-m-2 j-\frac{d}{2}\right)_{j}}{j(j-1)\left(5-m-2 j-\frac{d}{2}\right)_{j-2}}\left(\|x\|^{2}-1\right)^{2} P_{j-2, \ell}^{2, m}(\mathbf{x}) .
$$

The following formulas are very useful for the numerical analysis (see [22]):

$$
\begin{aligned}
& P_{j, \ell}^{-2, n}(\mathbf{x})=P_{j, \ell}^{0, n}(\mathbf{x})-\frac{2(2 n+d-4)}{2 n+d-6} P_{j-1, \ell}^{0, n-2}(\mathbf{x})+\frac{2 n+d-2}{2 n+d-6} P_{j-2, \ell}^{0, n-4}(\mathbf{x}), \quad j \geq 2, \\
& \Delta P_{j, \ell}^{-2, n}(\mathbf{x})=(2 n+d-4)(2 n+d-2) P_{j-1, \ell}^{0, n-2}(\mathbf{x}), \quad j \geq 2 .
\end{aligned}
$$

Thus, $X_{N}$ can be denoted by

$$
X_{N}=\left\{P_{j, \ell}^{-2, n}: 4 \leq n \leq N, 2 \leq j \leq \frac{n}{2}, 1 \leq \ell \leq a_{n-2 j}^{d}\right\} .
$$

Therefore, from (27), (28), and the property of orthogonal polynomials in the unit sphere, we have the following result (see $[22,26])$.

Lemma 4.1 We have the following formulas:

$$
\left\langle\Delta P_{j, \ell}^{-2, n}, \Delta P_{j^{\prime}, \ell^{\prime}}^{-2, n^{\prime}}\right\rangle=d(2 n+d-2)^{2}(2 n+d-4) b_{d}^{0} \delta_{n, n^{\prime}} \delta_{j, j^{\prime}} \delta_{\ell, \ell^{\prime}},
$$




$$
\begin{aligned}
& \left\langle P_{j, \ell}^{-2, n}, P_{j^{\prime}, \ell^{\prime}}^{-2, n^{\prime}}\right\rangle \\
& =b_{d}^{0} \delta_{\ell, \ell^{\prime}} \times \begin{cases}\frac{d}{2 n+d}+\frac{4 d(2 n+d-4)}{(2 n+d-6)^{2}}+\frac{d(2 n+d-2)^{2}}{(2 n+d-6)^{2}(2 n+d-8)}, & j=j^{\prime}, n=n^{\prime}, \\
-\frac{2 d}{2 n+d-2}-\frac{2 d(2 n+d+2)}{(2 n+d-6)(2 n+d-2)}, & j=j^{\prime}-1, n=n^{\prime}-2, \\
\frac{d(2 n+d+6)}{(2 n+d+2)(2 n+d)}, & j=j^{\prime}-2, n=n^{\prime}-4,, \\
-\frac{2 d}{2 n+d-6}-\frac{2 d(2 n+d-2)}{(2 n+d-6)(2 n+d-10)}, & j=j^{\prime}+1, n=n^{\prime}+2, \\
\frac{d(2 n+d-2)}{(2 n+d-6)(2 n+d-8)}, & j=j^{\prime}+2, n=n^{\prime}+4, \\
0, & \text { otherwise. }\end{cases}
\end{aligned}
$$

Thus, the unknown functions can be rewritten as follows:

$$
u_{N}=\sum_{n=4}^{N} \sum_{j=2}^{\lfloor(n) / 2\rfloor} \sum_{\ell=1}^{a_{n-2 j}^{d}} \widehat{u}_{j, \ell}^{n} P_{j, \ell}^{-2, n}
$$

with the coefficients $\left\{\widehat{u}_{j, \ell}^{n}\right\}$ to be determined. Then the Legendre-Galerkin approximation scheme (10) is simplified into a system of algebraic eigenvalue equations:

$$
A_{j, \ell}^{n} \widehat{u}_{j, \ell}^{n}=\lambda_{N} B_{j, \ell}^{n} \widehat{u}_{j, \ell}^{n} .
$$

It is easily known from Lemma 4.1 that the stiff matrix $A_{j, \ell}^{n}$ is essentially diagonal and the mass matrix $B_{j, \ell}^{n}$ is hepta-diagonal such that equation (30) can be efficiently solved.

\section{Numerical experiments}

In the following, two numerical tests are provided for computing eigenvalues of (1)-(2) on disk and ball, respectively, which is operated in MATLAB. Their numerical results are shown in Table 1 and Table 2.

It is easily seen from Table 1 that the results have at least ten-digit accuracy with $N \geq 15$. From numerical results, it is shown that $\lambda_{1}$ is a simple eigenvalue, but $\lambda_{2}$ and $\lambda_{4}$ are all eigenvalues with multiplicity 2 .

It is easily seen from Table 2 that the results have at least ten-digit accuracy with $N \geq 20$. From numerical results, we know that $\lambda_{1}$ also is a simple eigenvalue, but $\lambda_{2}$ and $\lambda_{5}$ are eigenvalues with multiplicity 3 and 5 , respectively.

Table 1 The approximate eigenvalues on the unit disk $\mathbf{B}^{2}$ in $\mathbf{R}^{2}$

\begin{tabular}{llll}
\hline $\mathbf{N}$ & $\boldsymbol{\lambda}_{\mathbf{1}}$ & $\boldsymbol{\lambda}_{\mathbf{2}}$ & $\boldsymbol{\lambda}_{\mathbf{4}}$ \\
\hline 10 & 104.3631056 & 452.0074329 & 1216.451916 \\
15 & 104.3631056 & 452.0045101 & 1216.407600 \\
20 & 104.3631056 & 452.0045101 & 1216.407600 \\
\hline
\end{tabular}

Table 2 The approximate eigenvalues on unit ball $\mathrm{B}^{3}$ in $\mathbf{R}^{3}$

\begin{tabular}{llll}
\hline $\mathbf{N}$ & $\boldsymbol{\lambda}_{\mathbf{1}}$ & $\boldsymbol{\lambda}_{\mathbf{2}}$ & $\boldsymbol{\lambda}_{\mathbf{4}}$ \\
\hline 10 & 237.7210683 & 769.9765197 & 1818.29231 \\
15 & 237.7210675 & 769.9634832 & 1818.167926 \\
20 & 237.7210675 & 769.9634832 & 1818.167924 \\
25 & 237.7210675 & 769.9634832 & 1818.167924 \\
\hline
\end{tabular}


By comparing the relative errors of numerical solutions, we found that the convergence rates of numerical solutions approximate $O\left(10^{-12}\right)$ when $N \geq 20$, which is consistent with those obtained for the theoretical case. It is also shown that finding the approximate solutions for the biharmonic eigenvalue equations with spectral-Galerkin method is computationally very effective.

\section{Conclusions}

In this study, we have developed a high accuracy numerical method by means of the spectral theory of compact operator for biharmonic eigenvalue equations on a spherical domain. By employing the orthogonal spherical polynomials approximation and the spectral theory of compact operator, we have derived the error estimates of approximate eigenvalues and eigenfunctions. We also, respectively, provided two numerical experiments in the two-dimensional case and three-dimensional cases to verify that our method is very effective. While we have restricted our attention in this study to the cases of biharmonic eigenvalue problems with constant coefficient. Whereas the approach presented in this article can be extended to biharmonic eigenvalue problems with variable coefficients.

Competing interests

The author declares to have no competing interests.

Author's contributions

The author wrote, read, and approved the final manuscript.

Acknowledgements

This research was supported by National Science Foundation of China grant 11271127.

Received: 1 February 2016 Accepted: 5 February 2016 Published online: 29 February 2016

References

1. Canuto, C: Eigenvalue approximations by mixed methods. RAIRO. Anal. Numér. 12(1), $27-50$ (1978)

2. Ishihara, K: On the mixed finite element approximation for the buckling of plates. Numer. Math. 33(2), 195-210 (1979)

3. Rannacher, R: Nonconforming finite element methods for eigenvalue problems in linear plate theory. Numer. Math. 33(1), 23-42 (1979)

4. Sun, J: Iterative methods for transmission eigenvalues. SIAM J. Numer. Anal. 49(5), 1860-1874 (2011)

5. An, J, Shen, J: Spectral approximation to a transmission eigenvalue problem and its applications to an inverse problem. Comput. Math. Appl. 69(10), 1132-1143 (2015)

6. Argyris, JH, Fried, I, Scharpf, DW: The tuba family of plate elements for the matrix displacement method (tuba family of plate elements for matrix displacement method based on polynomial functions for deflections of triangular elements under bending/trib/). Aeronaut. J. 72, 701-709 (1968)

7. Sun, J: A new family of high regularity elements. Numer. Methods Partial Differ. Equ. 28(1), 1-16 (2012). doi:10.1002/num.20601

8. Oh, HS, Davis, C, Jeong, JW: Meshfree particle methods for thin plates. Comput. Methods Appl. Mech. Eng. 209, 156-171 (2012)

9. Davis, CB: A partition of unity method with penalty for fourth order problems. J. Sci. Comput. 60(1), 228-248 (2014)

10. Adini, A, Clough, RW: Analysis of plate bending by the finite element method. NSF Report USA, Grant 7337 (1960)

11. Morley, LSD: The triangular equilibrium element in the solution of plate bending problems. Aeronaut. Q. 19, 149-169 (1968)

12. Shi, ZC: Error estimates of Morley element. Math. Numer. Sin. 12(2), 113-118 (1990)

13. Ciarlet, PG, Raviart, PA: A mixed finite element method for the biharmonic equation. In: Proceedings of Symposium on Mathematical Aspects of Finite Elements in PDE, pp. 125-145 (1974)

14. Babuška, I, Osborn, J, Pitkäranta, J: Analysis of mixed methods using mesh dependent norms. Math. Comput. 35(152), 1039-1062 (1980)

15. Monk, P: A mixed finite element method for the biharmonic equation. SIAM J. Numer. Anal. 24(4), 737-749 (1987)

16. Nazarov, S, Plamenevsky, BA: Elliptic Problems in Domains with Piecewise Smooth Boundaries. De Gruyter, Berlin (1994)

17. Engel, G, Garikipati, K, Hughes, TJR, Larson, MG, Mazzei, L, Taylor, RL: Continuous/discontinuous finite element approximations of fourth-order elliptic problems in structural and continuum mechanics with applications to thin beams and plates, and strain gradient elasticity. Comput. Methods Appl. Mech. Eng. 191(34), 3669-3750 (2002)

18. Brenner, SC, Sung, LY: $C^{0}$ interior penalty methods for fourth order elliptic boundary value problems on polygonal domains. J. Sci. Comput. 22(1-3), 83-118 (2005)

19. Chen, Q, Monk, P: Introduction to applications of numerical analysis in time domain computational electromagnetism. In: Frontiers in Numerical Analysis-Durham 2010, pp. 149-225 (2012) 
20. Brenner, SC, Monk, P, Sun, J: C interior penalty Galerkin method for biharmonic eigenvalue problems. In: Spectral and High Order Methods for Partial Differential Equations ICOSAHOM 2014. Lecture Notes in Computational Science and Engineering, vol. 106, pp. 3-15. Springer, Berlin (2015). doi:10.1007/978-3-319-19800-2

21. Adams, RA: Sobolev Spaces. Academic Press, New York (1975)

22. Li, H, Xu, Y: Spectral approximation on the unit ball. SIAM J. Numer. Anal. 52(6), 2647-2675 (2014)

23. John, E, Osborn, J: Spectral approximation for compact operators. Math. Comput. 29(131), $712-725$ (1975)

24. Babuška, I, Osborn, J: Eigenvalue problems. In: Handbook of Numerical Analysis, vol. II, pp. 641-784. Elsevier, Amsterdam (1991). doi:10.1016/S1570-8659(05)80042-0

25. $\mathrm{Bi}, \mathrm{H}$, Ren, S, Yang, Y: Conforming finite element approximations for a fourth-order Steklov eigenvalue problem. Math. Probl. Eng. 2011, 873152 (2011). doi:10.1155/2011/873152

26. Shen, J, Tang, T, Wang, LL: Spectral Methods, Algorithms, Analysis and Applications. Springer, Berlin (2011)

Submit your manuscript to a SpringerOpen ${ }^{\circ}$ journal and benefit from:

- Convenient online submission

- Rigorous peer review

Immediate publication on acceptance

- Open access: articles freely available online

- High visibility within the field

- Retaining the copyright to your article

Submit your next manuscript at $>$ springeropen.com 